\title{
Surgical Removal of a Live Worm by Stereotactic Targeting in Cerebral Sparganosis
}

\author{
-Case Report- \\ Misato NOBAYASHI, Hidehiro HiRABAYASHI, Toshisuke SAKAKI, \\ Fumihiko NishIMURA*, Hiroshi FUKUI**, Shigeaki ISHIZAKA*, \\ and Masahide YOSHIKAWA* \\ Department of Neurosurgery, * Department of Parasitology, and \\ ${ }^{* *}$ Third Department of Internal Medicine, Nara Medical University, Nara
}

\begin{abstract}
A 64-year-old man presented with generalized tonic clonic convulsion followed by weakness of the right lower extremity. He had a medical history of hypertension, hyperlipidemia, and right cerebellar infarction. Computed tomography (CT) showed a small high density nodule with an enhanced perifocal low density area in the left occipital lobe. $T_{1}$-weighted magnetic resonance (MR) imaging showed a ringshaped and partial string-like nodule with enhancement by gadolinium. $\mathbf{T}_{2}$-weighted MR imaging showed the white matter of the left occipital lobe as high intensity. CT and MR imaging seemed to indicate metastatic brain tumors, although cortical atrophy and ventricular dilation were recognized. Left parietal craniotomy was performed under stereotactic targeting to obtain a definitive diagnosis. During manipulation at the center of the targeted lesion, a white, tape-like body was found and recognized to be a live worm. Serological testing revealed strong immunopositivity against Spirometra mansoni. The infection route in the present case was probably through eating raw chicken meat. Cerebral sparganosis is extremely rare but should be considered in the differential diagnosis of metastatic brain tumors, especially in endemic areas.
\end{abstract}

Key words: cerebral sparganosis, stereotaxis, Spirometra mansoni

\section{Introduction}

Sparganosis is a parasitic infection caused by a sparganum, the plerocercoid larva of Spirometra mansoni, which most commonly invade the subcutaneous tissue or muscle. Cerebral sparganosis is rare, and the clinical and radiological findings mimic other diseases, especially metastatic brain tumor. We treated a patient with cerebral sparganosis using a stereotactic targeting system to remove a live worm.

\section{Case Report}

A 64-year-old Japanese man presented with generalized tonic clonic convulsion followed by weakness of the right lower extremity. The patient had a medical history of hypertension, hyperlipidemia, and right cerebellar infarction, but recent simple partial seizures were well controlled by administration of antiepileptic agent. Routine hematological and biochemical investigations showed no abnormalities, with no eosinophilia or leukocytosis. Computed tomography (CT) showed a small high density nodule with an enhanced perifocal low density area in the left occipital lobe (Fig. 1). $\mathrm{T}_{1}$-weighted magnetic resonance (MR) imaging showed a ring-shaped and partial string-like nodule with enhancement by gadolinium (Fig. 2A, B). $\mathrm{T}_{2}$-weighted MR imaging showed the white matter of the left occipital lobe as high intensity (Fig. 2C), suggesting perifocal edema. However, the left lateral ventricle was not initially compressed, and was rather dilated on follow-up MR imaging. Cerebral

Received May 2, 2005; Accepted October 12, 2005

Author's present address: M. Nobayashi, M.D., Medical Center for Emergency and Critical Care, Nara Prefectural Nara Hospital, Nara, Japan. 


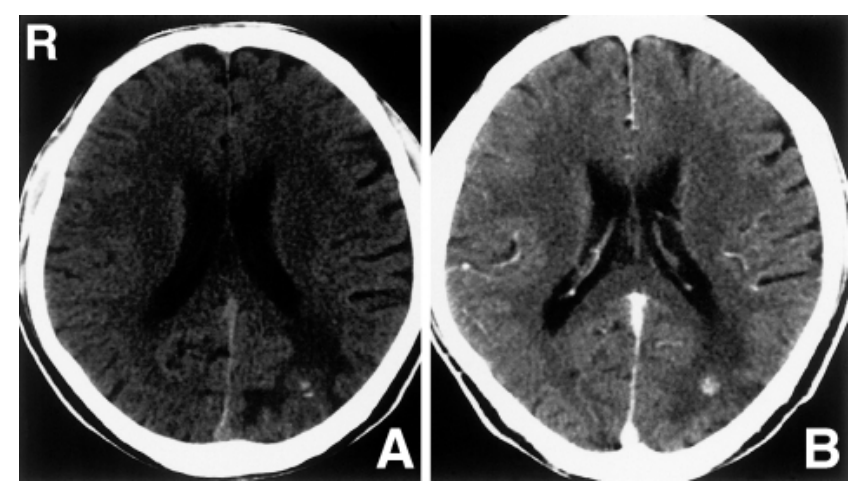

Fig. 1 Computed tomography scans showing a small high density nodule in the left occipital lobe (A), with enhancement by contrast medium (B).
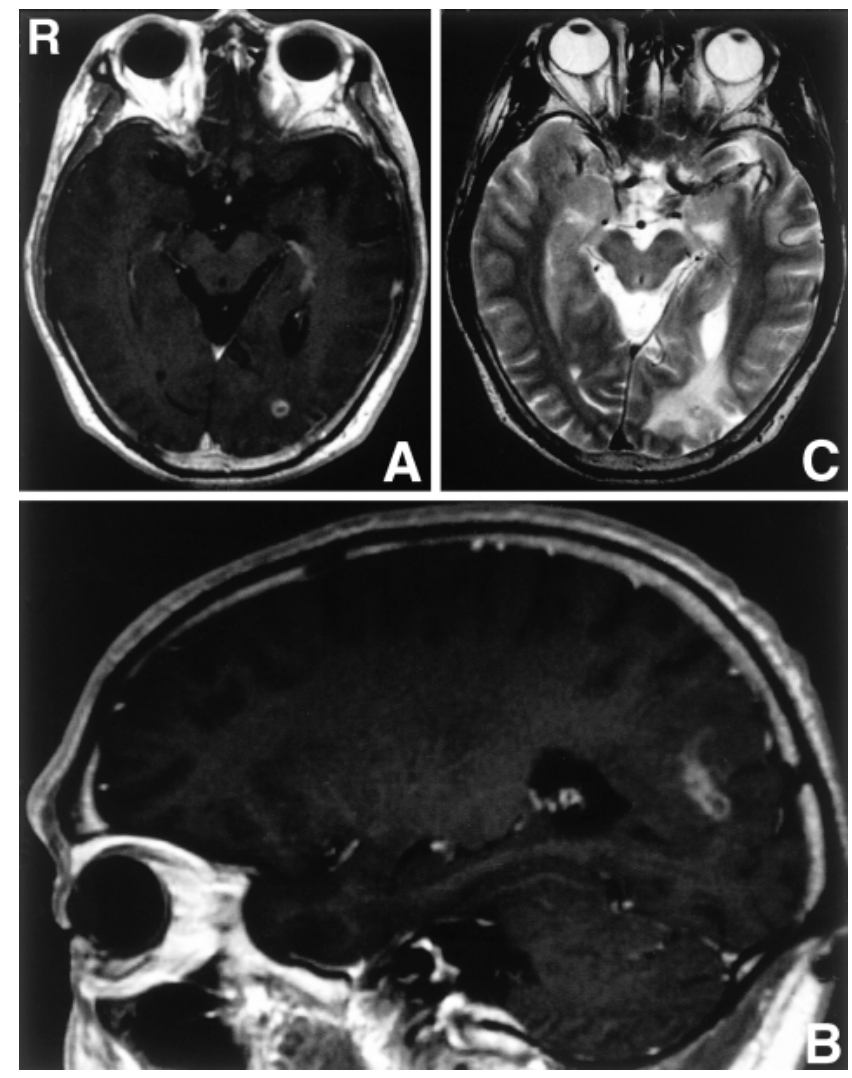

Fig. $2 T_{1}$-weighted magnetic resonance (MR) images showing a ring-shaped (A) and partial string-like nodule (B) with gadolinium enhancement. $T_{2}$-weighted $M R$ image showing a high intensity area in the occipital lobe associated with adjacent ventricular dilation (C).

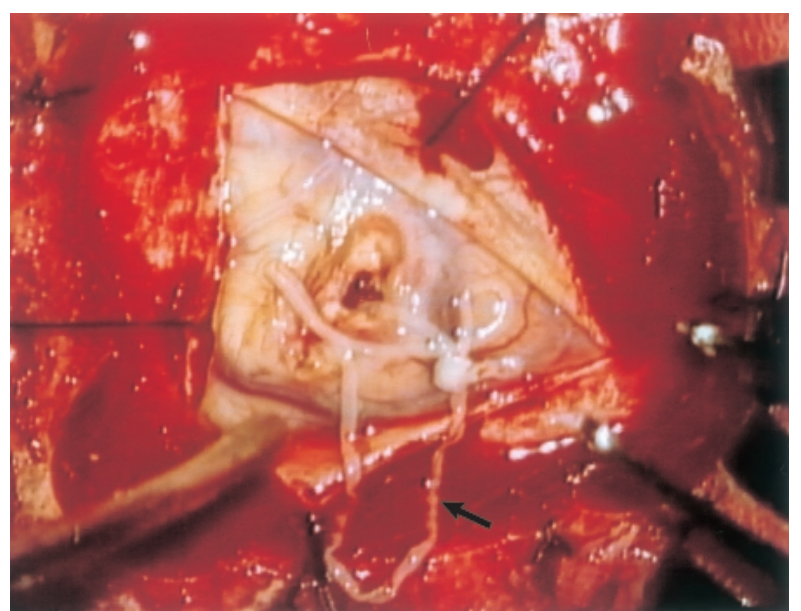

Fig. 3 Intraoperative photograph showing the live worm of Spirometra mansoni (arrow).

angiography showed normal findings, except for right vertebral artery occlusion, reflecting his past history of right cerebellar infarction. We suspected a metastatic brain tumor, but there was no history of malignant disease and a series of investigations for metastases found no evidence of another lesion.

A left parietal craniotomy was performed using a Cosman-Roberts-Wells stereotactic system for targeting the small and intraparenchymal nodule on March 1, 2002. The surface of the brain was relatively edematous, with no abnormal changes such as petechiae or punctate lesion. Intraoperative histological examination of the brain around the nodule revealed only gliosis. Manipulation of the aspirator and forceps at the center of the targeted lesion found a white, tape-like body (Fig. 3). A live worm was removed carefully and completely using forceps, and closer observation showed a cleft-like invagination in the head of parasite. Enzyme-linked immunosorbent assay (ELISA) on serum and cerebrospinal fluid revealed a strong positivity against Spirometra mansoni.

\section{Discussion}

Humans are considered to be an accidental intermediate host of Spirometra mansoni. Transmission occurs through drinking fresh water containing Cyclops, ingesting raw or inadequately cooked flesh from an infected second intermediate host, such as frogs, snakes, and chickens, or applying the flesh of an infected host as a poultice to an open wound. The infection route in the present case was probably through eating raw chicken meat, which the patient did approximately once a month before the convul- 
Table 1 Reports of successful removal of live worm in cerebral sparganosis

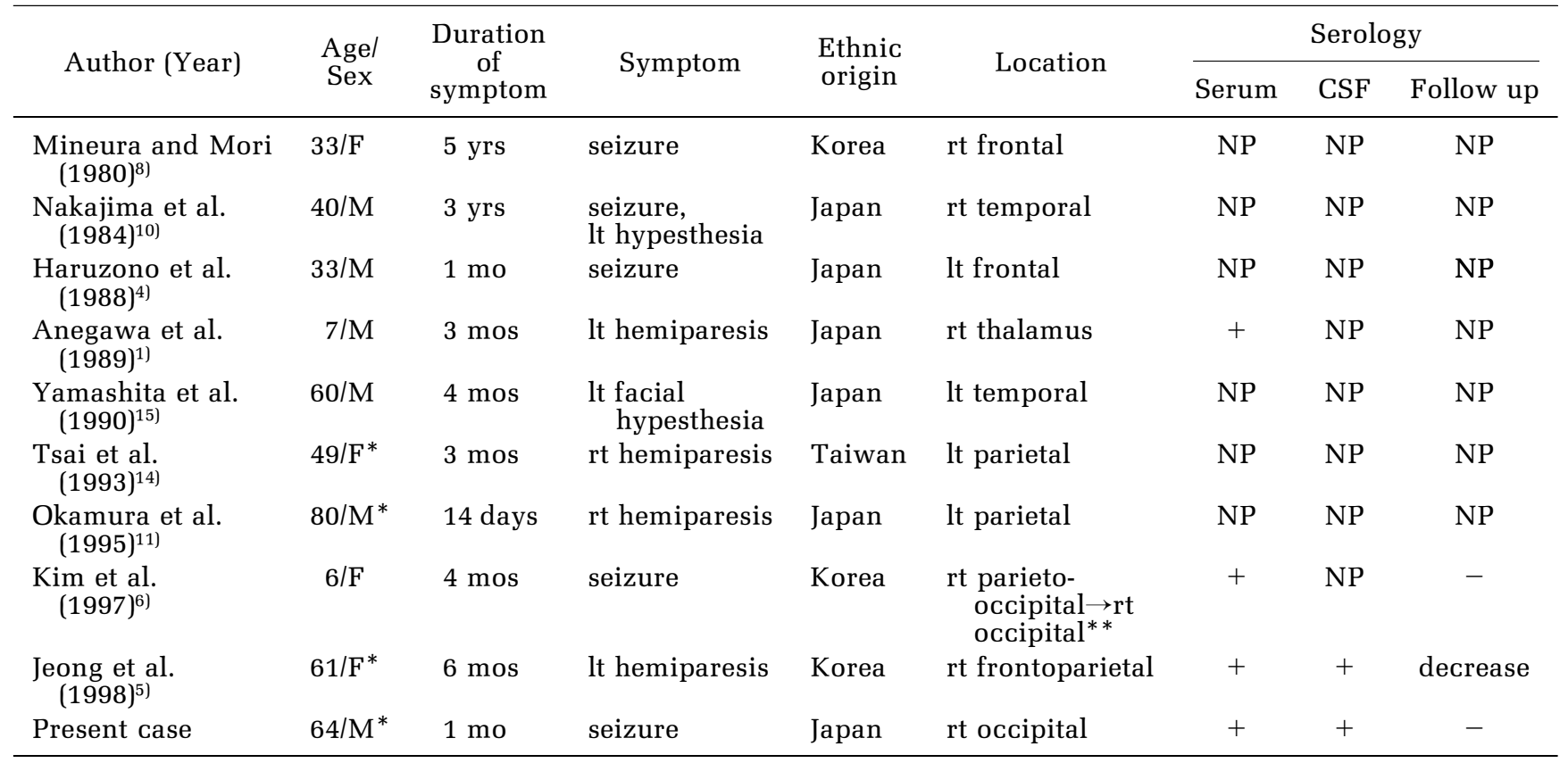

${ }^{*}$ Removal by stereotactic targeting. ${ }^{* *}$ Observed changes by neuroimaging. CSF: cerebrospinal fluid, NP: not performed, + or - : presence or absence of seropositivity for Spirometra mansoni.

sion attack.

Cerebral sparganosis was first reported in $1918,{ }^{12)}$ and most cases have been reported from Asia, including Korea and Japan..$^{1,3-6,8,10,11,15)}$ The route of infection to the central nervous system is not yet known, but the sparganum may unintentionally migrate through the foramina of the skull base and vertebral column along the loose connective tissue around the vessels or nerves. A live worm was removed in nine previous cases (Table 1). . $^{1,4-6,8,10,11,14,15)}$

Reports of cerebral sparganosis have been increasing, because of recent developments in neuroimaging techniques including $\mathrm{CT}^{2)}$ and $\mathrm{MR}$ imaging $^{7,9)}$ as well as serological studies. ${ }^{1,5-7,13)}$ The main CT findings consist of white matter hypodensity with adjacent ventricular dilation, irregular or nodular enhanced lesion, and small punctate calcification. ${ }^{2)}$ MR imaging findings consist of widespread white matter degeneration with cortical atrophy and ipsilateral ventricular dilation, mixed intensity lesion (low in the central and high in the peripheral region on $\mathrm{T}_{2}$-weighted images), irregular dense enhancement of central foci, and changes in the location and shape of the enhanced lesion in follow-up studies. The ELISA test is very sensitive and essential for the diagnosis of this parasitic disease. $^{7)}$

CT and MR imaging findings of cerebral sparganosis mimic those of metastatic brain tumor, usually appearing as an enhanced nodule with perifocal edema. Cerebral sparganosis is extremely rare but should be considered in the differential diagnosis of metastatic brain tumors, especially in endemic areas.

\section{References}

1) Anegawa S, Hayashi T, Ozuru K, Kuramoto S, Nishimura K, Shimizu T, Hirata M: Sparganosis of the brain. J Neurosurg 71: 287-289, 1989

2) Chang KH, Chi JG, Cho SY, Han MH, Han DH, Han MC: Cerebral sparganosis: analysis of 34 cases with emphasis on CT features. Neuroradiology 34: 1-8, 1992

3) Cummings TJ, Madden JF, Gray L, Friedman AH, McLendon RE: Diagnostic neuroradiology: Parasitic lesion of the insula suggesting cerebral sparganosis: case report. Neuroradiology 42: 206-208, 2000

4) Haruzono A, Niiro M, Hirahara K, Asakura T: [A case of sparganosis mansoni cerebri as an epileptogenic lesion]. No Shinkei Geka 16(5 Suppl): 603-606, 1988 (Jpn, with Eng abstract)

5) Jeong SC, Bae JC, Hwang SH, Kim HC, Lee BC: Cerebral sparganosis with intracerebral hemorrhage: a case report. Neurology 50: 503-506, 1998

6) Kim CY, Cho BK, Kim IO, Hwang YS, Wang KC: Cerebral sparganosis in a child. Pediatr Neurosurg 26: 103-106, 1997

7) Kim DG, Paek SH, Chang KH, Wang KC, Jung HW, Kim HJ, Chi JG, Choi KS, Han DH: Cerebral sparga- 
nosis: clinical manifestations, treatment, and outcome. J Neurosurg 85: 1066-1071, 1996

8) Mineura K, Mori T: Sparganosis of the brain. J Neurosurg 52: 588-590, 1980

9) Moon WK, Chang KH, Cho SY, Han $\mathrm{MH}$, Cha $\mathrm{SH}$, Chi JG, Han MC: Cerebral sparganosis: MR imaging versus CT features. Radiology 188: 751-757, 1993

10) Nakajima H, Mitsui T, Suzuki M, Takei K, Ishida $Y$, Kamo H: [Cerebral sparganosis. Case report]. Neurol Med Chir (Tokyo) 24: 427-432, 1984 (Jpn, with Eng abstract)

11) Okamura T, Yamamoto $M$, Ohta K, Matsuoka T, Uozumi T: Cerebral sparganosis mansoni. Case report. Neurol Med Chir (Tokyo) 35: 909-913, 1995

12) Takeuchi K: [A case with plerocercoid which is parasitic in the brain]. Nippon Byori Gakkai Kaishi 7: 611-620, 1918 (Jpn)

13) Tan TY, Lui CC, Chen HJ, Liou CW: Cerebral sparganosis: case report. Changgeng Yi Xue Za Zhi 22: 287-292, 1999

14) Tsai MD, Chang CN, Ho YS, Wang ADJ: Cerebral sparganosis diagnosed and treated with stereotactic techniques. Report of two cases. J Neurosurg 78: 129-132, 1993

15) Yamashita K, Akimura T, Kawano K, Wakuta Y, Aoki H, Gondou T: Cerebral sparganosis mansoni. Report of two cases. Surg Neurol 33: 28-34, 1990

Address reprint requests to: M. Nobayashi, M.D., Medical Center for Emergency and Critical Care, Nara Prefectural Nara Hospital, 1-30-1 Hiramatsu-cho, Nara 631-0846, Japan.

e-mail: nobarin@naramed-u.ac.jp 